\title{
Non-Gaussian Fluctuations near the QCD Critical Point
}

\author{
M. A. Stephanov \\ Department of Physics, University of Illinois, Chicago, Illinois 60607, USA
}

(Received 30 September 2008; published 20 January 2009)

\begin{abstract}
We study the effect of the QCD critical point on non-Gaussian moments (cumulants) of fluctuations of experimental observables in heavy-ion collisions. We find that these moments are very sensitive to the proximity of the critical point, as measured by the magnitude of the correlation length $\xi$. For example, the cubic central moment of multiplicity $\left\langle(\delta N)^{3}\right\rangle \sim \xi^{4.5}$ and the quartic cumulant $\left\langle(\delta N)^{4}\right\rangle_{c} \sim \xi^{7}$. We estimate the magnitude of critical point contributions to non-Gaussian fluctuations of pion and proton multiplicities.
\end{abstract}

DOI: 10.1103/PhysRevLett.102.032301

PACS numbers: 25.75.Nq, 05.70.Jk, 25.75.Gz

Introduction.-Mapping the QCD phase diagram is one of the fundamental goals of heavy-ion collision experiments. The QCD critical point is a distinct feature of the phase diagram, the existence of which is a ubiquitous property of QCD models [1] based on chiral dynamics. Locating the point using first-principles lattice calculations is a formidable challenge. Recent progress and results are encouraging, but much work needs to be done to understand and constrain systematic errors [2]. If the critical point is located in the region accessible to heavy-ion collision experiments, it can be discovered experimentally. The search for the critical point is planned at the Relativistic Heavy Ion Collider at Brookhaven National Laboratory, the Super Proton Synchrotron (SPS) at CERN, and the future Facility for Antiproton and Ion Research at Gesellschaft für Schwerionenforschung.

This Letter focuses on the experimental observables needed to locate the critical point in heavy-ion collisions. Locating the point requires a scan of the phase diagram, by varying the initial collision energy $\sqrt{s}$. The characteristic signature is the nonmonotonic behavior, as a function of $\sqrt{s}$, of the experimental observables sensitive to the proximity of the critical point to the point where freeze-out occurs for a given $\sqrt{s}[3,4]$.

The characteristic feature of a critical point is increase and divergence of fluctuations. Most fluctuation measures discussed to date can be related to quadratic variances of event-by-event observables, such as particle multiplicities, net charge, baryon number, particle ratios, or mean transverse momentum in the event [5] (with few exceptions [6]). Typically, the singular contribution to quadratic variances induced by the proximity of the critical point is proportional to approximately $\xi^{2}$, where $\xi$ is the correlation length which, in the idealized thermodynamic limit, would diverge at the critical point [4]. The magnitude of $\xi$ is limited trivially by the system size but most stringently by the finite-time effects due to critical slowing down $[4,7]$. The observation [7] that the correlation length may reach at most the value of 2-3 fm, compared to its "natural" value of $1 \mathrm{fm}$, may make discovering the critical nonmonotonic contribution to such fluctuation measures a challenging task, if the measures depend on $\xi$ too weakly.

Higher order as well as quadratic moments of fluctuations have been studied on the lattice using Taylor expansion around $\mu_{B}=0$ and in QCD models [2,8]. How sensitive are such higher moments to the proximity of the critical point?

In this Letter, we point out that higher, non-Gaussian, moments of the fluctuations are much more sensitive to the proximity of the critical point than the commonly employed measures based on quadratic moments. We explore this observation quantitatively, calculating the critical contribution to selected fluctuation observables.

We emphasize at the outset that only the critical contribution to these observables is addressed here. Other effects contributing to non-Gaussian moments are beyond the scope of this Letter (see also concluding discussion).

Illustration.-The main point can be illustrated using a description of the fluctuations based on the probability distribution of an order parameter field - any field which, by quantum numbers, can mix with the critical mode $\sigma$ the mode developing infinite correlation length at the critical point.

We choose the maximum of the probability distribution to be at $\sigma=0$. The probability distribution $P[\sigma]$ can be written as

$$
P[\sigma] \sim \exp \{-\Omega[\sigma] / T\},
$$

where $\Omega$ is the effective action (free energy) functional for the field $\sigma$, which can be expanded in powers of $\sigma$ as well as in the gradients:

$$
\Omega=\int d^{3} x\left[\frac{1}{2}(\nabla \sigma)^{2}+\frac{m_{\sigma}^{2}}{2} \sigma^{2}+\frac{\lambda_{3}}{3} \sigma^{3}+\frac{\lambda_{4}}{4} \sigma^{4}+\cdots\right] .
$$

Near the critical point, $m_{\sigma} \ll T$, so the mode $\sigma$ can be treated as a classical field.

Calculating 2-point correlator $\langle\sigma(x) \sigma(0)\rangle$, we find that the correlation length $\xi=m_{\sigma}^{-1}$. For correlation functions of the zero-momentum mode $\sigma_{0} \equiv \int d^{3} x \sigma(x) / V$, we find 


$$
\begin{aligned}
& \kappa_{2}=\left\langle\sigma_{0}^{2}\right\rangle=\frac{T}{V} \xi^{2} ; \quad \kappa_{3}=\left\langle\sigma_{0}^{3}\right\rangle=\frac{2 \lambda_{3} T}{V} \xi^{6} \\
& \kappa_{4}=\left\langle\sigma_{0}^{4}\right\rangle_{c} \equiv\left\langle\sigma_{0}^{4}\right\rangle-\left\langle\sigma_{0}^{2}\right\rangle^{2}=\frac{6 T}{V}\left[2\left(\lambda_{3} \xi\right)^{2}-\lambda_{4}\right] \xi^{8} .
\end{aligned}
$$

The critical point is characterized by $\xi \rightarrow \infty$. The central observation in this Letter is that the higher moments (cumulants) $\kappa_{3}$ and $\kappa_{4}$ diverge with $\xi$ much faster than the quadratic moment $\kappa_{2}$.

To be precise, the correlators scale slightly differently than Eqs. (3) suggest, e.g., $\left\langle\sigma_{0}^{2}\right\rangle \sim \xi^{2-\eta}$. Since the anomalous dimension $\eta \approx 0.04$ is very small, the difference between the actual asymptotic scaling and Eqs. (3) is discernible only for very large values of $\xi$, irrelevant in the context of this study. More importantly, the parameters $\lambda_{3}$ and $\lambda_{4}$ also scale with $\xi$ [see Eq. (12)].

Of course, the fluctuations of the critical mode are not measured directly in heavy-ion collision experiments. These fluctuations do, however, influence fluctuations of multiplicities, momentum distributions, ratios, etc., of observed particles, such as pions or protons, to which the critical mode couples [4]. The purpose of this Letter is to determine the magnitude of these effects.

Critical contribution to experimental observables.-We shall now estimate the effect of the critical point fluctuations on the observables such as the pion multiplicity fluctuations. Using a similar approach, it should be straightforward to construct corresponding estimates for such observables as charge, proton number, transverse momentum fluctuations, etc., as well as to take into account acceptance cuts.

We shall focus on the most singular contribution, proportional to a power of the correlation length $\xi$. This contribution can be found using an intuitive picture described in Ref. [4]: One considers a joint probability distribution for the occupation numbers $n_{p}$ of observed particles (e.g., pions) together with the value of the critical mode field $\sigma$ (more precisely, its zero-momentum mode $\sigma_{0}$ ), the latter treated as classical. Because of coupling of the critical mode of the type $\sigma \pi \pi$, the fluctuations of the occupation numbers receive an additional contribution, proportional to the corresponding correlation functions (moments) of the fluctuations of $\sigma_{0}$ given by Eq. (3). In this Letter, however, it will be more convenient to use instead the more formal diagrammatic method of Ref. [9].

Cubic cumulant.-The 3-particle correlator receives the following most singular contribution from the $\sigma$ fluctuations, given by the diagram in Fig. 1:

$$
\left\langle\delta n_{p_{1}} \delta n_{p_{2}} \delta n_{p_{3}}\right\rangle_{\sigma}=\frac{2 \lambda_{3}}{V^{2} T}\left(\frac{G}{m_{\sigma}^{2}}\right)^{3} \frac{v_{p_{1}}^{2}}{\omega_{p_{1}}} \frac{v_{p_{2}}^{2}}{\omega_{p_{2}}} \frac{v_{p_{3}}^{2}}{\omega_{p_{3}}} .
$$

Subscript $\sigma$ indicates that only the critical mode contribution is considered. As in Refs. [4,9], we denoted $\sigma \pi \pi$ coupling by $G$ and introduced a shorthand notation for the variance of the occupation number distribution: $v_{p}^{2}=$ $\bar{n}_{p}\left(1 \pm \bar{n}_{p}\right)$, where the " + " is for the Bose particles.

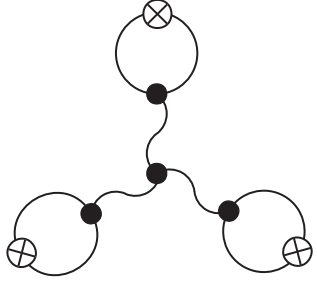

FIG. 1. Diagrammatic representation of the contribution to the three-particle correlator from the critical mode $\sigma$. Wavy lines represent propagators of the $\sigma$ field, each contributing factor $1 / m_{\sigma}^{2}$, and crossed circles represent insertions of $\delta n_{p}$ into the correlator Eq. (4)—see Ref. [9] for details.

Since the total multiplicity is just the sum of all occupation numbers and thus

$$
\delta N=\sum_{p} \delta n_{p}
$$

the cubic moment of the pion multiplicity distribution is given by

$$
\left\langle(\delta N)^{3}\right\rangle=V^{3} \int_{p 1} \int_{p 2} \int_{p 3}\left\langle\delta n_{p_{1}} \delta n_{p_{2}} \delta n_{p_{3}}\right\rangle,
$$

where $\int_{p} \equiv \int d^{3} \boldsymbol{p} /(2 \pi)^{3}$. Since $\left\langle(\delta N)^{3}\right\rangle$ scales as $V^{1}$, it is convenient to normalize it by the mean total multiplicity $\bar{N}$, which scales similarly. Thus we define

$$
\omega_{3}(N) \equiv \frac{\left\langle(\delta N)^{3}\right\rangle}{\bar{N}}
$$

and find

$$
\omega_{3}(N)_{\sigma}=\frac{2 \lambda_{3}}{T} \frac{G^{3}}{m_{\sigma}^{6}}\left(\int_{p} \frac{v_{p}^{2}}{\omega_{p}}\right)^{3}\left(\int_{p} \bar{n}_{p}\right)^{-1} .
$$

Quartic cumulant.-The leading contribution to the connected 4-particle correlator is given by the sum of two types of diagrams in Fig. 2:

$$
\begin{aligned}
\left\langle\delta n_{p_{1}} \delta n_{p_{2}} \delta n_{p_{3}} \delta n_{p_{4}}\right\rangle_{c, \sigma}= & \frac{6}{V^{3} T}\left[2\left(\frac{\lambda_{3}}{m_{\sigma}}\right)^{2}-\lambda_{4}\right]\left(\frac{G}{m_{\sigma}^{2}}\right)^{4} \\
& \times \frac{v_{p_{1}}^{2}}{\omega_{p_{1}}} \frac{v_{p_{2}}^{2}}{\omega_{p_{2}}} \frac{v_{p_{3}}^{2}}{\omega_{p_{3}}} \frac{v_{p_{4}}^{2}}{\omega_{p_{4}}} .
\end{aligned}
$$

The quartic cumulant of multiplicity fluctuations is given by

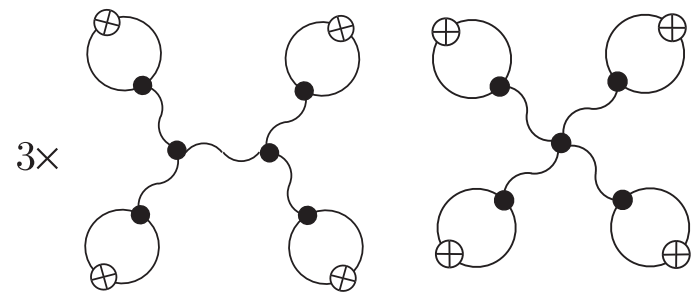

FIG. 2. Diagrammatic representation of the critical mode contribution to the connected four-particle correlator. Same notation as in Fig. 1. 


$$
\left\langle(\delta N)^{4}\right\rangle_{c}=V^{4} \int_{p_{1} p_{2} p_{3} p_{4}}\left\langle\delta n_{p_{1}} \delta n_{p_{2}} \delta n_{p_{3}} \delta n_{p_{4}}\right\rangle_{c} .
$$

This cumulant also scales as $V^{1}$ in thermodynamic limit. As in Eq. (7) we define a ratio whose $V \rightarrow \infty$ limit is finite: $\omega_{4}(N) \equiv\left\langle(\delta N)^{4}\right\rangle_{c} / \bar{N}$, and find

$$
\omega_{4}(N)_{\sigma}=\frac{6}{T}\left[2 \frac{\lambda_{3}^{2}}{m_{\sigma}^{2}}-\lambda_{4}\right] \frac{G^{4}}{m_{\sigma}^{8}}\left(\int_{p} \frac{v_{p}^{2}}{\omega_{p}}\right)^{4}\left(\int_{p} \bar{n}_{p}\right)^{-1} .
$$

Estimate of the effect.-In order to estimate the magnitude of the effect, we need to estimate the values of the coupling constants $\lambda_{3}$ and $\lambda_{4}$. The main uncertainty in the estimate will come, however, from the uncertainty of the value of $G$, which enters in a large power. This constant is known only roughly - the estimate was made in Ref. [4]. Therefore, a crude estimate for $\lambda$ 's suffices.

Near the critical point, both $\lambda_{3}$ and $\lambda_{4}$ vanish with a power of $\xi$ given by (neglecting $\eta \ll 1$ ):

$$
\lambda_{3}=\tilde{\lambda}_{3} T(T \xi)^{-3 / 2} \quad \text { and } \quad \lambda_{4}=\tilde{\lambda}_{4}(T \xi)^{-1},
$$

where dimensionless couplings $\tilde{\lambda}_{3}$ and $\tilde{\lambda}_{4}$ are universal, and for the Ising universality class they have been measured (see, e.g., Ref. [10] for a review). $\tilde{\lambda}_{3}$ varies from 0 to about 8 depending on the direction of approach to the critical point (crossover or first-order transition side). The coupling $\tilde{\lambda}_{4}$ varies from about 4 to about 20. Since the freeze-out occurs somewhere between these two extremes (as illustrated in Fig. 3), we shall pick some midrange values for our estimates. The main point is the strong dependence of the effect on $\xi$.

Putting together estimates of $\lambda, G$ (from [4]), and $\xi$ (from [7]), we find for pions at $T \approx 120 \mathrm{MeV}$ [in full acceptance, for a single pion species; see also Eq. (20)]:

$$
\begin{gathered}
\omega_{3}\left(N_{\pi}\right)_{\sigma} \approx 1\left(\frac{\tilde{\lambda}_{3}}{4}\right)\left(\frac{G}{300 \mathrm{MeV}}\right)^{3}\left(\frac{\xi}{3 \mathrm{fm}}\right)^{9 / 2}, \\
\omega_{4}\left(N_{\pi}\right)_{\sigma} \approx 12\left(\frac{2 \tilde{\lambda}_{3}^{2}-\tilde{\lambda}_{4}}{50}\right)\left(\frac{G}{300 \mathrm{MeV}}\right)^{4}\left(\frac{\xi}{3 \mathrm{fm}}\right)^{7} .
\end{gathered}
$$

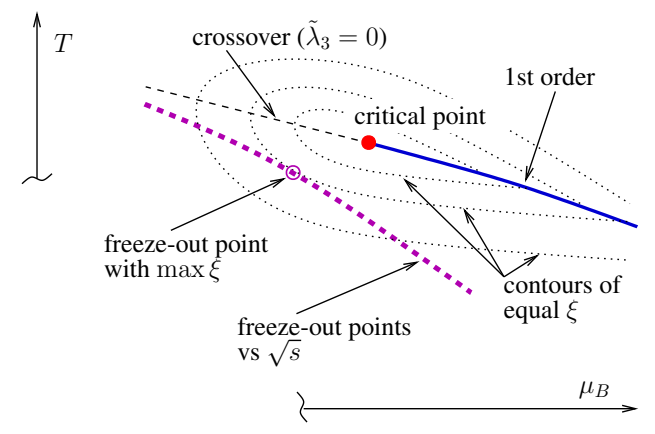

FIG. 3 (color online). Illustration of the possible relative position of the critical point and the locations of the freeze-out points for different values of the initial collision energy $\sqrt{s}$ in a heavy-ion collision.
Because of large powers of the coupling $G$, which is known only poorly, the uncertainty in this result is significantly larger than that of similar estimates of the quadratic moments of fluctuations [4].

The most significant feature of this result is the strong dependence on $\xi$ which makes the cubic and quartic cumulants very sensitive signatures of the critical point.

Another example: proton multiplicity fluctuations.-The above analysis carries over to the proton multiplicity fluctuations. The fluctuations of the net proton number are a good proxy to the baryon number fluctuations, whose magnitude, proportional to the baryon number susceptibility, must diverge at the critical point [11]. Here, for simplicity, we shall present the results for the proton only multiplicity, which is easier to measure.

To adapt an equation such as Eq. (4) to protons, one needs to substitute $G$ with the coupling $g$ of the critical mode $\sigma$ to protons (times the mass $m_{p}$ of the proton): $G \rightarrow$ $g m_{p}$. The estimate for this coupling can be taken from the sigma model to be roughly $g \approx m_{p} / f_{\pi} \approx 10$. The variance of the occupation number distribution is $v_{p}^{2}=\bar{n}_{p}(1-$ $\left.\bar{n}_{p}\right)$ and $\bar{n}_{p}=\left(e^{\left(\omega_{p}-\mu_{B}\right) / T}+1\right)^{-1}$, where $\mu_{B}$ is baryochemical potential. There is also a factor of $2^{k-1}$ for $\omega_{k}$ [as in Eq. (20)] because of the proton spin degeneracy. Putting this together, one finds, e.g., for protons at SPS freeze-out conditions $\left(T, \mu_{B}\right) \approx(168,266) \mathrm{MeV}$ [12],

$$
\begin{gathered}
\omega_{3}\left(N_{p}\right)_{\sigma} \approx 6\left(\frac{\tilde{\lambda}_{3}}{4}\right)\left(\frac{g}{10}\right)^{3}\left(\frac{\xi}{1 \mathrm{fm}}\right)^{9 / 2}, \\
\omega_{4}\left(N_{p}\right)_{\sigma} \approx 46\left(\frac{2 \tilde{\lambda}_{3}^{2}-\tilde{\lambda}_{4}}{50}\right)\left(\frac{g}{10}\right)^{4}\left(\frac{\xi}{1 \mathrm{fm}}\right)^{7} .
\end{gathered}
$$

Note that the effect is much larger on the proton multiplicity fluctuations, compared to the pion multiplicity.

Similar to quadratic fluctuations [11], the exponents in Eqs. (15) and (16) agree (up to $\eta \ll 1$ ) with the critical exponents of the baryon number cumulants dictated by scaling and universality:

$$
\left\langle\left(\delta N_{B}\right)^{k}\right\rangle_{c}=V T^{k-1} \frac{\partial^{k} P\left(T, \mu_{B}\right)}{\partial \mu_{B}^{k}} \sim \xi^{k(5-\eta) / 2-3} .
$$

Mean transverse momentum.-From the expression for the correlators Eq. (4) or (9), one can similarly estimate the effect of the critical point on other observables, for example, higher moments of the fluctuation of mean transverse momentum $p_{T}$. For example, the cubic moment $\kappa_{3}\left(\delta p_{T}\right)$ of the mean $p_{T}$ distribution around the all-event mean $\bar{p}_{T}$ can be expressed as

$$
\begin{aligned}
\kappa_{3}\left(\delta p_{T}\right) \equiv & \left\langle\left(p_{T}-\bar{p}_{T}\right)^{3}\right\rangle \\
= & \sum_{\boldsymbol{p}_{1}, \boldsymbol{p}_{2}, \boldsymbol{p}_{3}}\left(\left[\boldsymbol{p}_{1}\right]_{T}-\bar{p}_{T}\right)\left(\left[\boldsymbol{p}_{2}\right]_{T}-\bar{p}_{T}\right) \\
& \times\left(\left[\boldsymbol{p}_{3}\right]_{T}-\bar{p}_{T}\right)\left\langle\delta n_{\boldsymbol{p}_{1}} \delta n_{\boldsymbol{p}_{2}} \delta n_{\boldsymbol{p}_{3}}\right\rangle
\end{aligned}
$$

and estimated using Eq. (4). Normalizing this variable 
similarly to the variable $F$ proposed in Ref. [4] removes $N$ scaling and makes it less sensitive to the effect of the flow ("blueshift" of momenta):

$$
F_{3} \equiv \frac{\bar{N} \kappa_{3}\left(p_{T}\right)}{v_{\text {inc }}^{3 / 2}\left(p_{T}\right)}
$$

where $v_{\text {inc }}\left(p_{T}\right)$ is the variance of the inclusive (singleparticle) $p_{T}$ distribution. We leave this to future work.

Comments and discussion.--It is worth noting that, even though the $\xi$ dependence of $\omega_{4}$ is stronger, its measurement involves subtraction of two contributions $\left\langle(\delta N)^{4}\right\rangle-$ $3\left\langle(\delta N)^{2}\right\rangle^{2}$, each of which is order $N$ times larger than their difference, which might dilute the signal-to-noise ratio in experimental measurement.

Since the freeze-out occurs, generically, somewhat off the crossover line, as illustrated in Fig. 3, one should expect the critical point contribution to fluctuations to be skewed. In this case, the deviations from the Gaussian shape are dominated by the cubic moment or $\omega_{3}$.

What is the sign of $\omega_{3}(N)_{\sigma}$ ? One can anticipate it by using the following, admittedly crude, argument. The skewness of the distribution of the order parameter near the critical point is a "shadow" of a second peak. This peak corresponds to the phase on the other side of the firstorder transition line (quark-gluon plasma phase at higher $T$ and $\mu_{B}$ in Fig. 3). This phase has higher entropy and baryon number; thus, fluctuations of these quantities must be skewed toward higher values: $\omega_{3}>0$. Since pion and proton numbers are rough proxies to the entropy and the baryon number, respectively, their skewness should be also positive.

What is the natural, background value one should expect for, e.g., $\omega_{3}$ ? For a gas of classical free particles (Poisson distribution), $\omega_{3}(N)=1$. Bose statistics increases this by a factor $\omega_{3}(N)_{\mathrm{BE}}=\overline{\left(1+n_{p}\right)\left(1+2 n_{p}\right)}$, e.g., approximately 1.3 for pions at $T=120 \mathrm{MeV}$.

More importantly, quantum statistics correlates only fluctuations of particles of the same species; thus, $\omega_{3}(N)_{\mathrm{BE}}$ is the same for all charge (i.e., $N=N_{\pi^{+}}+$ $N_{\pi^{-}}$) and single charge multiplicity fluctuations. In contrast, the critical point contribution correlates also $\pi^{+}$with $\pi^{-}$, thus making $\omega_{3} 4$ times larger for all charge vs single charge (for $\bar{N}_{\pi^{+}}=\bar{N}_{\pi^{-}}$):

$$
\omega_{k}\left(N_{\pi^{+}}+N_{\pi^{-}}\right)_{\sigma}=2^{k-1} \omega_{k}\left(N_{\pi^{+}}\right)_{\sigma} .
$$

Equation (20) can help separate critical point contribution from contributions due to quantum statistics.

It is important to note that other sources may and do contribute to the skewness and kurtosis: remnants of initial fluctuations, flow, and jets-to name just a few obvious contributors. Quantitative study of these effects might be necessary to unambiguously identify the critical point signal. This serves to emphasize that the energy scan of the QCD phase diagram is needed to separate such background contributions from the genuine critical point effect, the latter being the nonmonotonic function of the initial collision energy $\sqrt{s}$ as the critical point is approached and then passed. The fact that non-Gaussian moments have stronger dependence on $\xi$ than, e.g., quadratic moments makes those higher moments more sensitive signatures of the critical point.

This work is a direct consequence of discussions at the INT program "The QCD Critical Point." The author is grateful to the participants and especially to P. de Forcrand, S. Gupta, F. Karsch, V. Koch, J. Randrup, K. Rajagopal, K. Redlich, D. Son, and M. Tsypin for stimulating and useful discussions. The hospitality of the Institute of the Nuclear Theory at the University of Washington and partial support of the Department of Energy during the completion of this work is gratefully acknowledged. This work is supported by DOE Grant No. DE-FG0201ER41195.

[1] M. Asakawa and K. Yazaki, Nucl. Phys. A504, 668 (1989); A. Barducci, R. Casalbuoni, S. De Curtis, R. Gatto, and G. Pettini, Phys. Lett. B 231, 463 (1989); Phys. Rev. D 41, 1610 (1990); for further references and review see, e.g., M.A. Stephanov, Prog. Theor. Phys. Suppl. 153, 139 (2004); Int. J. Mod. Phys. A 20, 4387 (2005).

[2] Z. Fodor and S. D. Katz, J. High Energy Phys. 03 (2002) 014; S. Ejiri, C. R. Allton, S. J. Hands, O. Kaczmarek, F. Karsch, E. Laermann, and C. Schmidt, Prog. Theor. Phys. Suppl. 153, 118 (2004); R. V. Gavai and S. Gupta, Phys. Rev. D 71, 114014 (2005); arXiv:0806.2233 [Phys. Rev. D (to be published)]; P. de Forcrand and O. Philipsen, arXiv:hep-ph/0301209; for further references see reviews, e.g., C. Schmidt, Proc. Sci., LAT2006 (2006) 021; M. A. Stephanov, Proc. Sci. LAT2006 (2006) 024.

[3] M. A. Stephanov, K. Rajagopal, and E. V. Shuryak, Phys. Rev. Lett. 81, 4816 (1998).

[4] M. A. Stephanov, K. Rajagopal, and E. V. Shuryak, Phys. Rev. D 60, 114028 (1999).

[5] For review and references see, e.g., S. Jeon and V. Koch, arXiv:hep-ph/0304012.

[6] S. Mrowczynski, Phys. Lett. B 465, 8 (1999).

[7] B. Berdnikov and K. Rajagopal, Phys. Rev. D 61, 105017 (2000).

[8] R. V. Gavai and S. Gupta, Phys. Rev. D 72, 054006 (2005); S. Ejiri, F. Karsch, and K. Redlich, Phys. Lett. B 633, 275 (2006); B. Stokic, B. Friman, and K. Redlich, arXiv:0809.3129.

[9] M. A. Stephanov, Phys. Rev. D 65, 096008 (2002).

[10] M. M. Tsypin, Phys. Rev. Lett. 73, 2015 (1994); arXiv: hep-lat/9401034; arXiv:hep-lat/9601021.

[11] Y. Hatta and M. A. Stephanov, Phys. Rev. Lett. 91, 102003 (2003); 91, 129901(E) (2003).

[12] P. Braun-Munzinger, J. Stachel, J. P. Wessels, and N. Xu, Phys. Lett. B 365, 1 (1996). 